\title{
Endovascular Therapy: A Standard Treatment?
}

\author{
Jong S. Kim \\ Editor-in-Chief, Journal of Stroke
}

MR CLEAN, ESCAPE, EXTEND-IA, SWIFT PRIME, REVASCAT, THERAPY, THRACE: we are inundated by positive trials. In this issue of the Journal of Stroke (JOS), two important review papers are published. Dr. Ding nicely summarizes these results, ${ }^{1}$ while Dr. Yan and his colleagues emphasize the advancement of devices as one of the main reasons for the successful trials. ${ }^{2}$ Their messages are clear; endovascular therapy is helpful for treating stroke patients as long as appropriate patients are selected and good devices are used by skillful interventionalists.

These recent series of trials will change the landscape of the neurology world. Although countries such as Korea already have actively used endovascular therapy, the endovascular strategy will soon spread worldwide. It is expected that increasing numbers of young physicians will choose to become endovascular specialists, device companies will prosper, and endovascular societies will strengthen. Accordingly, there will be a rapid increase in the number of comprehensive stroke centers equipped with advanced imaging tools and endovascular specialists. Undoubtedly, these factors will benefit stroke patients. However, this also means that medical costs will increase dramatically.

Since excellent stroke centers armed with modern imaging tools and experienced interventionalists are costly even in affluent countries, such costs are likely to unacceptable in less affluent countries. Regardless of economic conditions, the costs will skyrocket globally as populations worldwide continue to age rapidly. In reality, the cost incurred by treating and preventing cerebrovascular diseases is already a significant burden to us; therefore, we must continue investigating less costly strategies.

Endovascular therapy developed as a result of the defects of intravenous (IV) tissue plasminogen activator (t-PA), which has limited efficacy in re-canalizing occluded large arteries, has a very short therapeutic time window ( $<4.5$ hours), and is associated with hemorrhagic complications unless administered early enough.

To resolve these problems, drugs have been developed that are potentially more effective and safe than IV t-PA. One candidate is desmoteplase, which unfortunately does not yet have proven benefit. Several drugs such as minocycline are now being examined to determine whether they can reduce bleeding complications when used with IV t-PA. These and other drugs under development may increase the efficacy and safety or prolong the therapeutic time window of IV thrombolysis. I suspect that they may ultimately replace endovascular therapy, a more costly and complicated way of treatment, in the future.

Although I fully agree with Drs. Ding and Yan, and am one of the most excited neurologists by these positive trials, I also worry that official acceptance of endovascular therapy as a "standard treatment" might prevent the proper examination of simpler, less expensive and potentially more effective treatments. We all know that potentially efficacious thrombolytics could not be tested in the early stage of stroke simply because IV t-PA has been designated a "standard treatment."

I hope my concern is groundless, and wish that you read this issue of the JOS comfortably.

\section{References}

1. Ding D. Endovascular mechanical thrombectomy for acute ischemic stroke: a new standard of care. J Stroke 2015;17:123126.

2. Balasubramaian A, Mitchell P, Dowling R, Yan B. Evolution of endovascular therapy in acute stroke: implications of device development. J Stroke 2015;17:127-137.

\footnotetext{
Correspondence: Jong S. Kim

Department of Neurology, Asan Medical Center, University of Ulsan

College of Medicine, 88 Olympic-ro 43-gil, Songpa-gu,

Seoul 138-736, Korea

Tel: +82-2-3010-3442, Fax: +82-2- 474-4691

E-mail: jongskim@amc.seoul.kr

The author has no financial conflicts of interest.
} 